\title{
Research on Modified SVM for Image classification in Remote Sensing
}

\author{
Chuankai Zhang ${ }^{1, a}$, Fangji Liang ${ }^{2, b}$ \\ ${ }^{1}$ Wuhan University, Wuhan 430072, China; \\ ${ }^{2}$ Shanghai Maritime University, Shanghai, 201306, China; \\ a625498689@qq.com, ${ }^{\mathrm{b}} 707537264 @ q q . c o m$
}

\begin{abstract}
Keywords: Image classification, support vector machines, artificial neural network, kappa
\end{abstract} coefficient.

\begin{abstract}
Image classification is based on the analysis in different time from the same area of two or more images, detect the feature in the region information changes over time. Remote sensing image classification has been widely used in such as the dynamic monitoring of forest resources monitoring, the change of land cover and use, agricultural resources survey, urban planning layout, environmental monitoring and analysis, assessment of natural disasters, geographic data update and military reconnaissance in the strategic objectives (such as roads, Bridges, airports) of dynamic monitoring and many other fields. SVM classifiers are most prominently used classifiers and they provide good accuracy. This research paper presents a modified SVM classifier by incorporating intelligence into the proposed system. Intelligence is provided by using an ANN architecture. The proposed SVM-ANN approach aims to reduce the impact of parameters in classification accuracy. In the training stage, the SVM is utilized to reduce the training samples for each of the available categories to their support vectors (SVs). The SVs from different categories are used as the training data of nearest neighbor classification algorithm in which the similarity measures or distance function is used to calculate the which class does the testing data belongs and which also reduce time consumption.
\end{abstract}

\section{Introduction}

Remote sensing becomes an integral part of human life especially with the advancements in science and technology. They provide essential information in areas requiring constant updates such as weather forecasting, temperature monitoring, classification of agricultural lands for cultivation, land erosion etc., Land use land cover information is also essential to many socio-economic and environmental applications, including urban and regional planning, natural resources conservation and management, etc. [1] [4] [7]. Remote sensing imagery, covering a large geographic area with high temporal frequency, offers a unique opportunity for deriving land use and land cover information through the process of image interpretation and classification. Remote sensing techniques are gaining more and more importance for land cover classification and urban analysis. The accuracy of pixel based classification approaches are affected by the increase in resolution of images and object based approaches are devised for improving the performance. The availability of high resolution satellite images have popularized the object based classification and literature suggests a great deal of advanced methodologies for the purpose. The spectral and spatial information can be combined to increase the separability between classes to yield higher classification accuracy. A general scheme of image classification is depicted in figure 1.

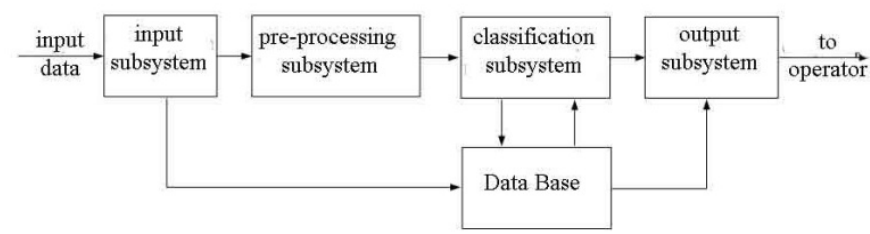

Figure 1. A general image classification model 
The input SAR (synthetic aperture radar) image is pre- processed using a suitable $\mathrm{m} \mathrm{x} \mathrm{m}$ window filter preferably a wiener filter to provide quality enhancement to the acquired image. The pre-processed image is then given to the classification kernel and based on the features extracted which are then compared with the features stored in the knowledge data base, the output provides the classification map. In remote sensing applications, the most commonly used classification sets include land green cover, water systems, vegetated land, non-cultivated land etc., the evaluation parameters include accuracy, precision, kappa coefficient.

\section{Associated works}

A survey on remote sensing classification have been done in a systematic manner including mostly intelligent classifiers under the scope of this section. A novel fuzzy-topology [12] integrated support vector machine (SVM) (FTSVM) classification method for remotely sensed images based on the standard SVM has also been presented in the past. Induced threshold fuzzy protocol is included into the standard SVM. Two different experiments were performed to evaluate the performance of the FTSVM technique, in assessment with standard SVM, maximum likelihood classifier (MLC), and fuzzy topology- integrated MLC. The FTSVM method performs better than the standard SVM and other methods in terms of classification exactness, thus providing a successful classification method for remotely sensed images. FTSVM when compared with the standard SVM, MLC, and FTMLC, the FTSVM obtains a comparatively high accuracy.

FSVM is used to enhance the SVM in reducing the effect of outliers and noises in data points and is suitable for applications, in which data points have un-modeled characteristics [11]. Combing the advantages of statistical learning framework and the fuzzy basis function inference system, An SVM-based fuzzy inference system [9] which provides reliable performance in the cases of classification and prediction. A discussion on unclassifiable regions for multiclass problems which were resolved using fuzzy LS-SVMs are presented in [10]. Fuzzy topology is generalized from ordinary topology by introducing the concept of membership value in a fuzzy set. A hybrid technique [3] utilizing combination of KPCA, SVM and GA algorithms has been presented and implemented. KPCA reduces the Eigen space input space features and features are classified using SVM. The SVM parameters are optimized by GA. However, the selection of parameters effects on the detection performance, when the difference between features are high, using the RBF kernel in the training phase, it produce a huge number of support vectors. Both of them are vital for achieving high performance of detection, intrusion and feature extraction.

A hypothetical [14] conversation and experimental analysis aimed at understanding and assessing the potentialities of SVM classifiers in hyper dimensional feature spaces. Thus, the considered dataset allow to identify the following three properties: 1) SVMs are much more effective than other conventional nonparametric classifiers (i.e., the RBF neural networks and the K-nun classifier) in terms of categorization accurateness, computational time, and constancy to parameter setting; 2) SVMs seem more effective than the traditional pattern recognition method that is based on the blend of a feature extraction/selection procedure and a conventional classifier.

The authors [13] have used a method for feature deleting at each time employ to SVM and ANN, after that the features are ranked and for five different classes in DARPA intrusion data, efficient features are demonstrated. But, since the unimportant and/or pointless inputs cause a complex problem, slower and less accurate detection results. Author used data in their own experiments that initiate from MIT's Lincoln Lab. This technique considered a benchmark for evaluating and creating an intrusion detection system by DARPA. Using the importance features gives the most significant performance as far as training time. Using 2 classifiers are time consuming and hard task to trigger them.

This paper is organized as follows. Section II describes the proposed methodology. Section III illustrates the experimentation results and analysis followed by conclusion in section IV. 


\section{Proposed work}

Let $\mathrm{I}_{0}$ and $\mathrm{I}_{1}$ be two co-registered optical images, composed of $\mathrm{N}$ pixels each and acquired over the same area at times $t_{0}$ and $t_{1}$, respectively $\left(t_{1}>t_{0}\right)$. We assume $I_{0}$ and $I_{1}$ to be single-channel images; the reformulation in the multichannel case is straightforward. Change detection is formalized as a binary hypothesis testing problem, by marking the "change" and "no-change" hypotheses as $\mathrm{H}_{1}$ and $\mathrm{H}_{0}$, respectively. Image differencing, which generates a difference image $\mathrm{D}$ by subtracting pixel-by-pixel the pixel intensities in $\mathrm{I}_{0}$ by the pixel intensities in $\mathrm{I}_{1}$.

\section{A. SVM Classifier}

The SVM is an independent and identically distributed classifier that does not consider interactions in the labels of adjacent data points but have the appealing generalization properties (Support vector machines (SVMs) are a supervised non-parametric statistical learning technique made on the underlying data distribution. In its original formulation [1] the method is presented with a set of labeled data instances and the SVM training algorithm aims to find a hyper plane that separates the dataset into a discrete predefined number of classes in a fashion consistent with the training examples. The term optimal separation hyper plane is used to refer to the decision boundary that minimizes misclassifications, acquired in the training phase. Learning means to the iterative process of finding a classifier with optimal decision boundary to separate the training patterns (in potentially high-dimensional space) and then to separate simulation data under the same configurations.

\section{B. ANN - SVM Classifier}

The architecture of our hybrid ANN-SVM model was designed by replacing the last output layer of the ANN model with an SVM classifier. For output units of the last layer in the ANN network, they are the estimated probabilities for the input sample. Each output probability is calculated by an activation function. The input of the activation function is the linear combination of the outputs from the previous hidden layer with trainable weights, plus a bias term. Looking at the output values of the hidden layer is meaningless, but only makes sense to the ANN network itself; however, these values can be treated as features for any other classifiers. Firstly, the normalized and centered input images are sent to the input layer, and the original ANN with the output layer is trained with several epochs until the training process converges. Then, the SVM with a Radial Basis Function (RBF) kernel replaces the output layer. The SVM takes the outputs from the hidden layer as a new feature vector for training. Once the SVM classifier has been well trained, it performs the recognition task and makes new decisions on testing images with such automatically extracted features.

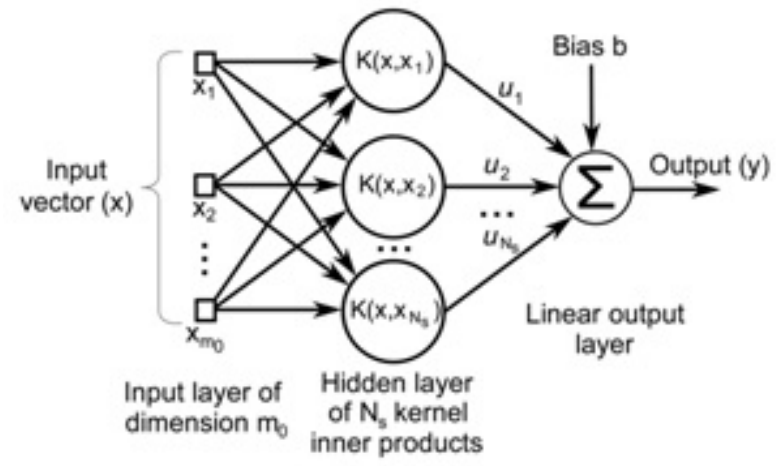

Figure 2. ANN 3 layer network architecture

\section{Results and discussion}

The experimentation has been done on an Intel I3 3.5GHz processor with input test images taken from Air change benchmark set 1 of Sztaki data base. The technique has been compared with the conventional Fuzzy and PCA based techniques. The dataset contains images from three data sets of 
optical aerial images provided by the Hungarian Institute of Geodesy Cartography \& Remote Sensing and Google Earth and corresponding Ground Truth change masks. All the images have been aligned as ortho-photos. The first data set - called Szada - contains co-registered images taken by FOMI in years 2000 and 2005. This test set consists of image pairs, covering in aggregate $9: 5 \mathrm{~km}^{2}$ area at $1.5 \mathrm{~m} /$ pixel resolution. The size of each image in the test set is $952 \times 640$ pixels). The second test set called Tiszadob - includes five image pairs from 2000 and 2007 (6:8km²) with similar size and quality parameters to Szada. Images of 2000 and 2005 were scanned on photo-films Hasselblad 500 EL/M) before digital scan.

\begin{tabular}{|l|l|l|l|}
\hline Extensibility & Circularity & Shape index & Form factor \\
\hline 0.846154 & 0.919866 & 2.953857 & 2.506628 \\
\hline 0.329442 & 0.573970 & 3.081526 & 0.596888 \\
\hline 0.827586 & 0.909718 & 2.894238 & 2.133048 \\
\hline 0.458763 & 0.677320 & 2.833953 & 1.322470 \\
\hline 0.800000 & 0.894427 & 2.683282 & 2.241996 \\
\hline 0.588235 & 0.766965 & 2.583073 & 1.823840 \\
\hline
\end{tabular}

Figure 3 Feature extraction for water bodies

Accuracy evaluation is to compare two images, and one of the pieces is to be evaluation of image, and the other is to assume that the picture is accurate reference map. Precision evaluation is very important for remote sensing classification, because the classification accuracy of RS image directly affects the mapping of the remote sensing image, management of report for the actual land environment and usefulness of other data analysis and rationality of the scientific research used these data. Two important parameters have been taken for evaluation of the proposed classification algorithm namely accuracy and kappa coefficient. Accuracy is a probabilistic parameter which is defined as one probability to each a random sample expressed that the region corresponding of the result of classification is in consistent with the actual type. Mathematically it could be presented as

Kappa analysis uses another discrete multivariate technology to overcome the above disadvantages. The evaluation index of Kappa analysis is called hat $K$.It is a statistics, a kind of index of determination of goodness of fit or accuracy between two picture. Based on the Kappa, we conduct finally choose the overall accuracy evaluation as the basis.

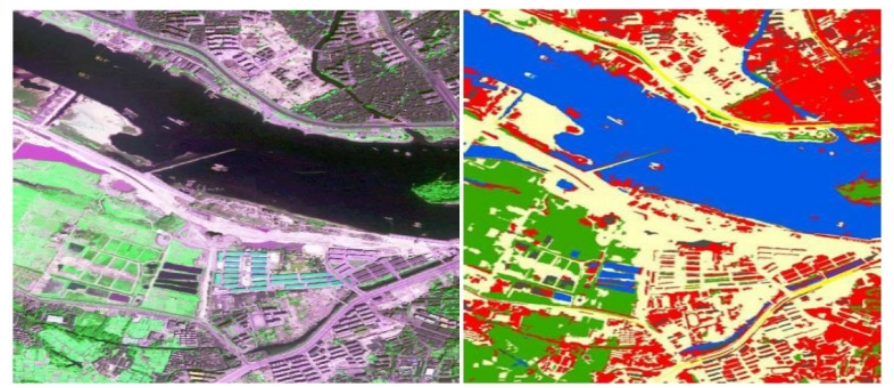

Figure 4 Input Quick bird image and its classification map

The images are up sampled over a wide range of magnification factors using various algorithms. However, for clear visual comparison, the images in this paper are generated with 2, 3, and $4 \times$ magnification. We quantify each algorithm's accuracy by computing the precision rate.

It could be seen that the proposed work provides a drastic reduction in the computation time. The accuracy results of the proposed work are also shown in table 2. The computation time is depicted in table 1. 
Table 1. Computation time comparison

\begin{tabular}{|l|l|l|}
\hline $\begin{array}{l}\text { S.N } \\
\text { o. }\end{array}$ & Algorithm & $\begin{array}{l}\text { Computation } \\
\text { time (s) }\end{array}$ \\
\hline 1. & Proposed work & 09.11 \\
\hline 2. & SVM - KNN & 11.58 \\
\hline 3. & SVM & 16.66 \\
\hline
\end{tabular}

Table 2. Accuracy result comparison

\begin{tabular}{|l|l|l|}
\hline $\begin{array}{l}\text { S.N } \\
\text { o. }\end{array}$ & Algorithm & $\begin{array}{l}\text { Accuracy } \\
\text { result (\%) }\end{array}$ \\
\hline 1. & Proposed work & 93.28 \\
\hline 2. & SVM - KNN & 89.75 \\
\hline 3. & SVM & 81.33 \\
\hline
\end{tabular}

\section{Conclusion}

Remote sensing image classification techniques are essential in deriving land use land cover information for socio-economic planning and environmental applications. Currently, spectral classifiers are still the dominant approaches for classifying remote sensing imagery due to their conceptual simplicity and easy implementation. Recently, an increasing number of researchers have realized the importance of spatio-contextual information in complementing spectral classifiers. Through conducting a comprehensive literature review on remote sensing classification methods, especially the spatio-contextual classification techniques, we have obtained several conclusions. By comparing with the results of the other traditional classification method, it can be see that the comprehensive use of multi-scale segmentation method and SVM with the texture and spectral characteristics for remote sensing image classification can achieve good effect. With the use of remote sensing image in wetland information extraction more and more widely, it is key question to use more accurate classification methods in the field of classification application. The method makes classification area relatively complete and the continuity between strong categories. In addition, the method based on multi-scale segmentation and SVM has strong operability and practicability for feature information.

\section{Reference}

[1] J.S. Xia,J. Chanussot,P.J Du,et al, “(Semi-) Supervised Probabilistic Principal Component Analysis for Hyper-spectral Remote Sensing Image Classification”, IEEE Journal of selected topics in applied earth observations and remote sensing, Vol. 7, No. 6, pp. 2225-2237, 2014.

[2] D. Amarsaikhan and M. Saandar, "Knowledge-Based Classification of Multisource Images of Mongolia”, Computational Science Applications, Vol. 1, No. 4, pp. 252-258, 2014.

[3] X.F. Song, Z. Duan, X.G. Jiang, "Comparison of artificial neural networks and support vector machine classifiers for land cover classification in Northern China using a SPOT-5 HRG image”, International Journal of Remote Sensing, Vol. 33, No. 10, pp. 3301-3320, 2012.

[4] C. Wang, A.Y. Shi, X. Wang et al, "A novel multi-scale segmentation algorithm for high resolution remote sensing images based on wavelet transform and improved JSEG algorithm", International Journal for Light and Electron Optics, Vol. 125, No. 19, pp. 5588-5595, 2014.

[5] S.Y. Zang, C. Zhang, L.J. Zhang, Y.H. Zhang, "Wetland Remote Sensing Classification Using Support Vector Machine Optimized With Genetic Algorithm”, Scientia Geogrphica Sinica, Vol. 32, No. 4, pp. 434-441, 2012. 
[6] Adjorlolo C., Mutanga O., "Integrating remote sensing and geostatistics to estimate woody vegetation in an African savanna. Journal of Spatial Science, Vo. 58, No. 2, pp. 305-322, 2013.

[7] Atkinson P.M., Naser D.K., "A geostatistically weighted k-NN classifier for remotely sensed imagery”, Geographical Analysis, Vol. 42, pp. 204-225, 2012.

[8] Bahria S., Essoussi N., Limam M., "Hyperspectral data classification using geostatistics and support vector machines. Remote Sensing Letters, Vol. 2, pp. 99-106, 2011.

[9] Comber A.J., "Geographically weighted methods for estimating local surfaces of overall, user and producer accuracies”, Remote Sensing Letters, Vol. 4, pp. 373-380, 2013.

[10] Dwivedi R.S., Kandrika S., Ramana K.V., "Comparison of Classifiers of Remote-Sensing Data for Land-Use/Land-Cover Mapping” Current Science, Vol. 86, pp. 328-335, 2012.

[11] Stathakis D, and Vasilakos A. "Comparison of computational intelligence based classification techniques for remotely sensed optical image classification”, IEEE Transaction on. Geoscience and Remote Sensing, vol. 44, issue 8, pp. 2305-2318, 2006.

[12] Hua Zhang, Wenzhong Shi, and Kimfung Liu "Fuzzy-Topology-Integrated Support Vector Machine for Remotely Sensed Image Classification”, IEEE Transactions on Geosciences And Remote Sensing, Vol. 50, No. 3, March 2012.

[13] Farid Melgani, Lorenzo Bruzzone "Classification of Hyper spectral Remote Sensing Images With Support Vector Machines”, IEEE Transactions on Geosciences And Remote Sensing, Vol. 42, No. 8, August 2004. 